\title{
Simulation of the lumbar spine as a multi-module paralel manipulator
}

\author{
M. Ceccarelli ${ }^{\mathrm{a}, *}$ R.C. Saltarelo ${ }^{\mathrm{b}}$ G. Carbone J.C.M. Carvalho $^{\mathrm{b}}$ \\ ${ }^{a}$ Laboratory of Robotics and Mechatronics, DiMSAT, University of Cassino, Cassino, Italy \\ ${ }^{\mathrm{b}}$ School of Mechanical Engineering, Federal University of Uberlandia, Campus Santa Monica, \\ Uberlandia/MG, Brazil
}

\begin{abstract}
In this paper a simulation of movements of lumbar spine is proposed by using a model with serially connected parallel manipulators. An analysis has been computed for the human spine structure and its movements, in order to simulate the motions and forces that actuate a spine specifically in the lumbar segment. A mechanical model has been designed with available identified parameters of human spine, by using characteristics of parallel manipulators and spring stiffness. This model is suitable to properly simulate the trunk behavior at macroscopic level but also the smooth behavior of intervertebral discs and actuating motions of muscles and tendons. Simulation results for spring actions and joints reaction forces can give an evaluation of the forces that intervertebral discs supports during motions of a real spine.
\end{abstract}

Keywords: Biomechanics, model of human spine, simulation

\section{Introduction}

A spine is a complex remarkable mechanical structure. Its main aim is to protect the spinal cord and nerve roots and to provide an incredible amount of flexibility to the trunk in supporting the body weight. A spine transmits the weight of the upper body to the pelvis and is subjected to internal forces whose magnitudes are many times the entire body weight. A spine consists of discrete bony elements called vertebras that are joined by passive ligaments restraints. They are kept separated by intervertebral discs and articulating joints, and they are dynamically controlled by muscular actions. A spine is broadly divided into five regions, namely cervical spine, thoracic spine, lumbar spine, sacrum, and coccyx. Each region has its own unique set of kinematic functions, pathologies, and treatments.

*Corresponding author: M. Ceccarelli, Laboratory of Robotics and Mechatronics, DiMSAT, University of Cassino, Via G. Di Biasio, 43. 03043 Cassino, Italy. E-mail: ceccarelli@unicas.it.
According to IFToMM a parallel manipulator is a manipulator that controls the motion of its endeffector by means of at least two kinematic chains going from the end-effector towards the frame, [2]. A parallel manipulator consists of a fixed base platform, connected to an end-effector platform by means of a number of legs. These legs often consist of an actuated prismatic joint within a link that is connected to the platforms through passive spherical and/or universal joints. Hence, the links feel only traction or compression, as a peculiarity which increases their position accuracy and allows a lighter construction [1]. Parallels manipulators can be modeled as closed kinematic chains in which the links are jointed to each other so that polygonal loops can be determined as kept during the motion. The kinematic behavior of a parallel manipulator depends in which space it will operate and therefore it can be planar or spatial [1].

The aim and contribution of the paper can be recognized in the model using parallel manipulators structures that can be useful to imitate the trunk 
behaviour at a macroscopic level. The simulation results are used both to characterize the human-like operation of the proposed model and to compare those internal reactions with those of a human spine. The parallel manipulator architecture has been thought convenient for modelling a human spine because it can give reference bodies as platforms that can be identified in specific parts of the human trunk and because it can focus on motion output of those reference bodies, but even by preserving the general structure of parallel systems in the musco-skeleton human spine. In addition, in the presented model the payload had been assumed as applied to the first platform in its center point in order to make possible a comparison with results that can be found in the literature. But using this kind of parallel structure a payload can be applied in any position and direction as well as a local momentum can be considered in order to analyze the spine behavior also when not in regular postures. Furthermore, the considered actuators, as assembled in the system, can simulate lateral movements of the spine in any desired direction. The considered springs and dampers simulate mainly damping and stiffness of vertebral disks but additional ones can be considered to better simulate muscles around the spine. Several other musculoskeletal models have been proposed in a quite riche literature both from technical and biomedical viewpoints, like for examples those listed in references for considering visualization aims of spine motion in $[8,15]$, a stiffness evaluation of a spine as serial connected bodies in $[6$, $9,13]$, stress analysis in spine behavior in [10-12, 14].

Modeling and motion simulations of a lumbar spine considered as a multi-module parallel manipulators are illustrated in this work. An analysis of the spine structure and spine motions have been made in order to simulate the human spine as specifically for the lumbar segment and to characterize the biomechanics of the spine motion with a numerical evaluation.

\section{The human spine}

The spine is a series of individual bones, which are the vertebrae, that are linked to each other to form the central axis of the skeletal structure of a human body. The flexibility of the spine comes from the relative mobility of vertebrae to each other, but its stability depends mainly on the muscles and ligaments. Although basically it is a skeletal body, the human spine can be considered as a composed structure from a practical point of view with its content and its annexes, which include the muscles, nerves, and veins. Its length is about two fifths of the total height of a human body. It consists of 24 mobile pre-sacral vertebrae (7 cervical, 12 thoracic and 5 lumbar). The five immediately below the lumbar vertebrae are fused in adults to form the sacrum. The four also lower vertebrae fuse to form the coccyx. The spine of an adult features four sagittal curves, namely they are cervical, thoracic, lumbar and sacral curves. Curvature is an important aspect of the functionality of human spine and the proposed model can include curvature inputs in the configurations of the whole spine model by using proper relative positioning and motion of the parallel manipulator units. In Fig. 1 the main structure and all regions of the spine are shown with their characteristic curvatures, [4].

Although anatomical characteristics of vertebrae can present regional variations along the spine, vertebrae have basic common morphology. A typical vertebra consists of a body, an arch, and vertebral processes. In Fig. 2 vertebral parts and basic geometrical dimensions are shown. The dimensions in Fig. 2(b) are an average from real values that were obtained with digitized CT images, as reported in, [7].

Intervertebral discs are elastic viscous fibrocartilagíneas cushions that form the main structure of joints between adjacent vertebral bodies. Typically, they consist of a core pulpous that is surrounded by a fibrous ring. In fibrous annulus, two portions can be identified, namely an external portion and an inner portion. The external portion is strongly anchored to the adjacent vertebral bodies, mixing with the longitudinal ligaments. It is the ligament portion of the fibrous annulus. The inner portion forms a dense envelope around the nucleus spheroidal pulpous. The core pulpous, which occupies the center of the disc, is white, bright and semi gelatinous. It is highly plastic and behaves as a viscous fluid. The basic regions and geometrical characteristics of the intervertebral discs are presented in Fig. 3. The dimensions in Fig. 3(b) are an average from of the real values that were obtained with digitized CT images as reported in, [7].

\section{Biomechanics of a spine}

The primary function of a spine consists in musculoskeletal support and mechanics. It provides longitudinal stiffness of a human body, by allowing 

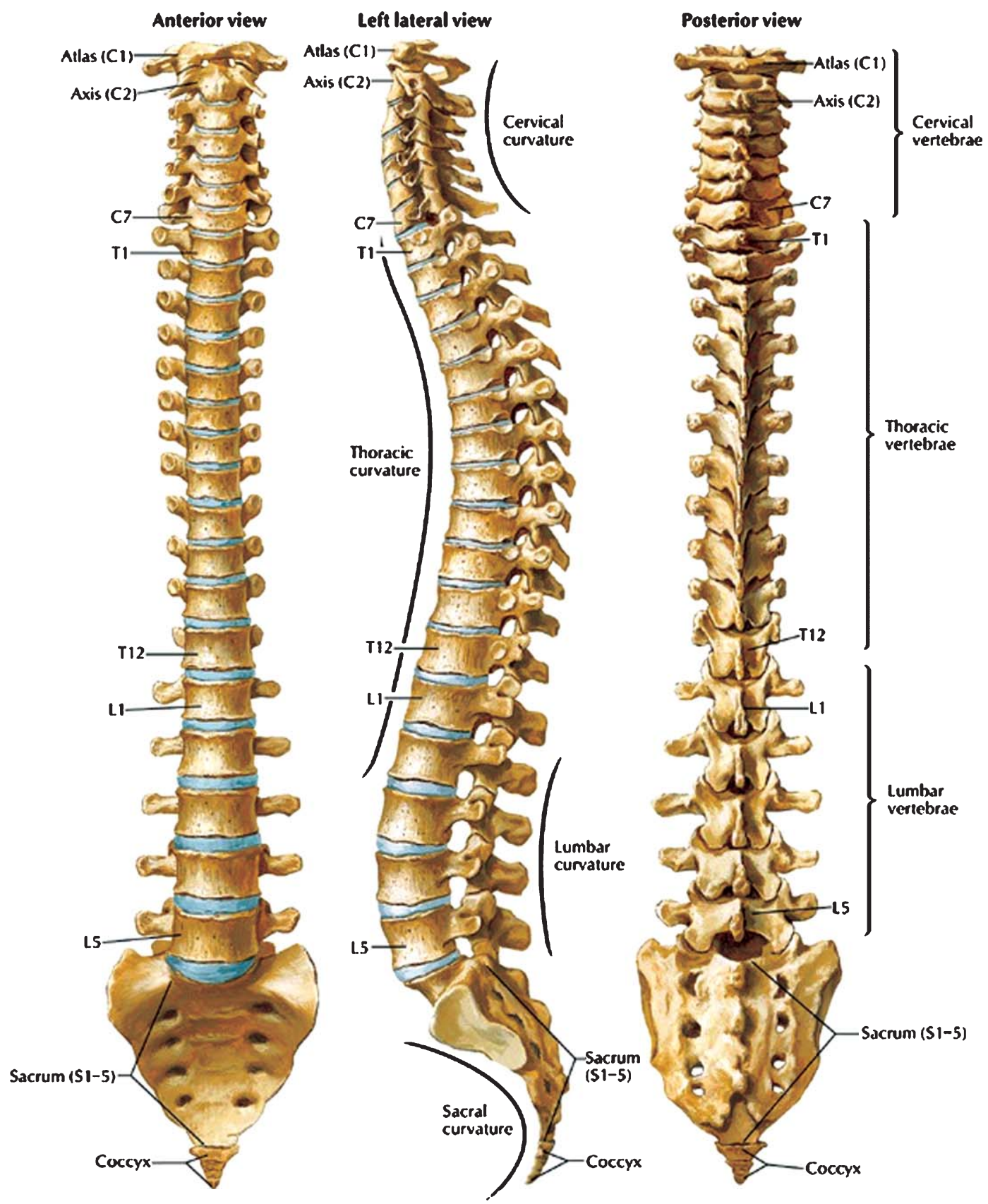

Fig. 1. Structure and regions of a human spine, [4].

movement between its parts. Secondly it constitutes a firm support of adjacent anatomical structures such as ribs and abdominal muscles by allowing the maintenance of body cavities with shape and size relatively constant. Other function of the spine is to allow the spinal cord gain access to distant parts of the trunk and limbs.

Motions of a human spine are characterized by the actuation planes that are the sagittal plane, coronal plane, and longitudinal plane. The most important 


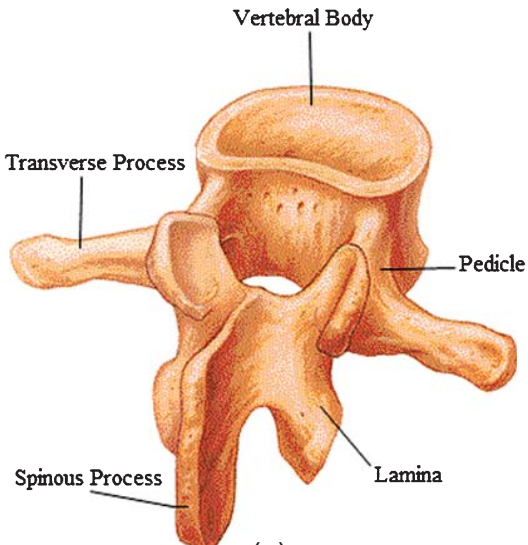

(a)

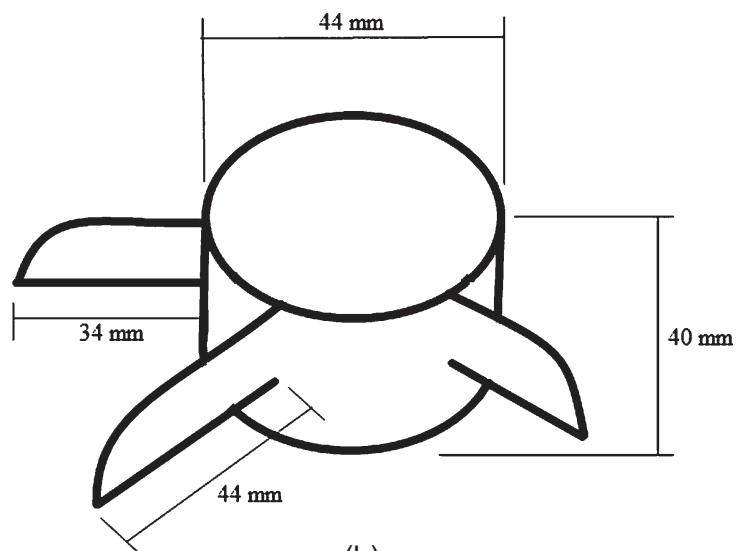

(b)

Fig. 2. A typical vertebra body: a) shape and components, [5]; b) a simplified model.

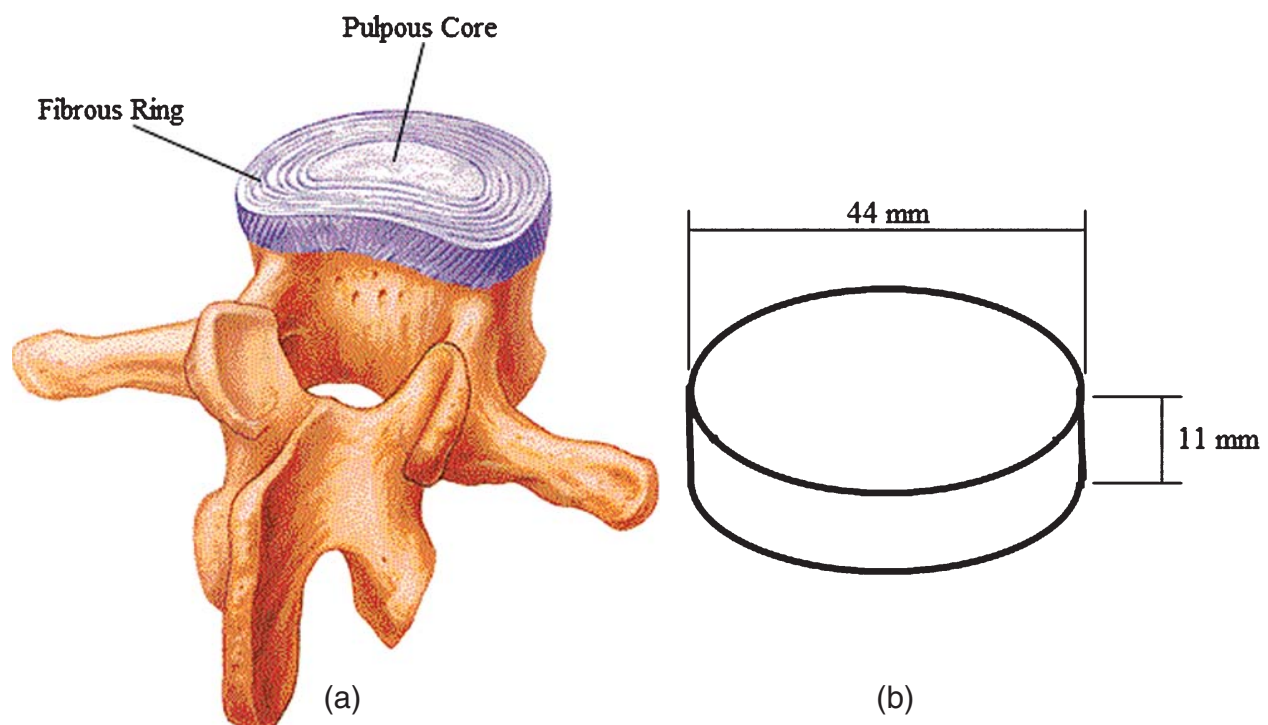

Fig. 3. The intervertebral disc: a) shape and components, [5]; b) a simplified model.

motions are flexion, extension, lateralization, and rotation.

The basic movements of the spine can be classified by using planes, as follows:

- In Sagittal plane: Flexion and Extension

- In Coronal plane: Right and Left Lateralization

- In Longitudinal plane: Rotation

These motions are illustrated in in Fig. 4, [4].

The motions of each segment of a spine have a different range for each type of movement but they can be modeled by rotations about the $\mathrm{X}, \mathrm{Y}$ and $\mathrm{Z}$ axis, as shown in Fig. 5.
These rotations give movements that can be classified by type as flexion, extension, lateralization, and rotation, as listed in Table 1. In this table the motions of flexion and extension of the thoracic segment is not considered since they are very small and of difficult measure, [4].

\section{A cad model for motion simulation}

The spine is a very complex structure of the human body that supports the upper part of it, so that simulating all solicitations, viscosity properties, 

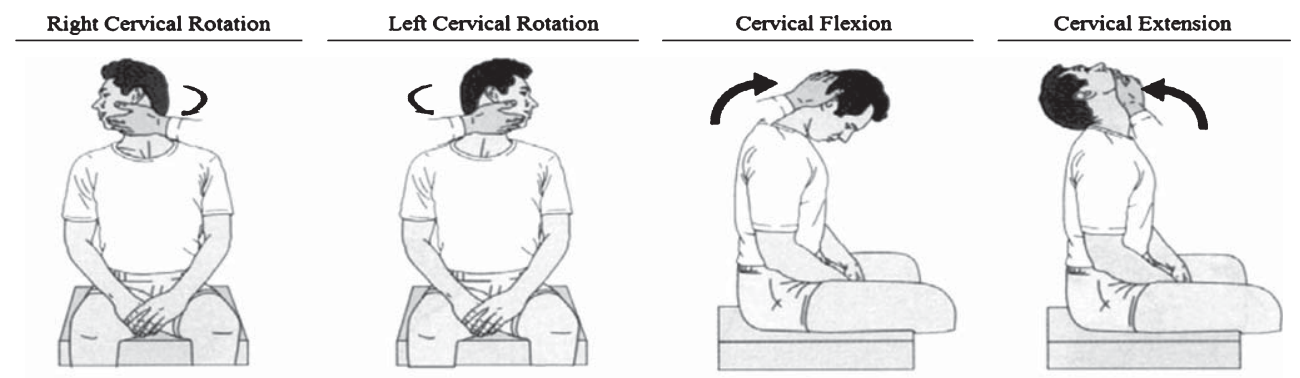

$\underline{\text { Right Cervical Lateralization }}$

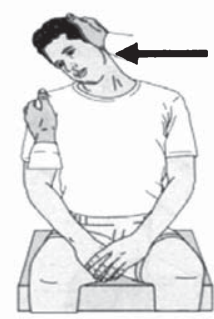

Left Cervical Lateralization
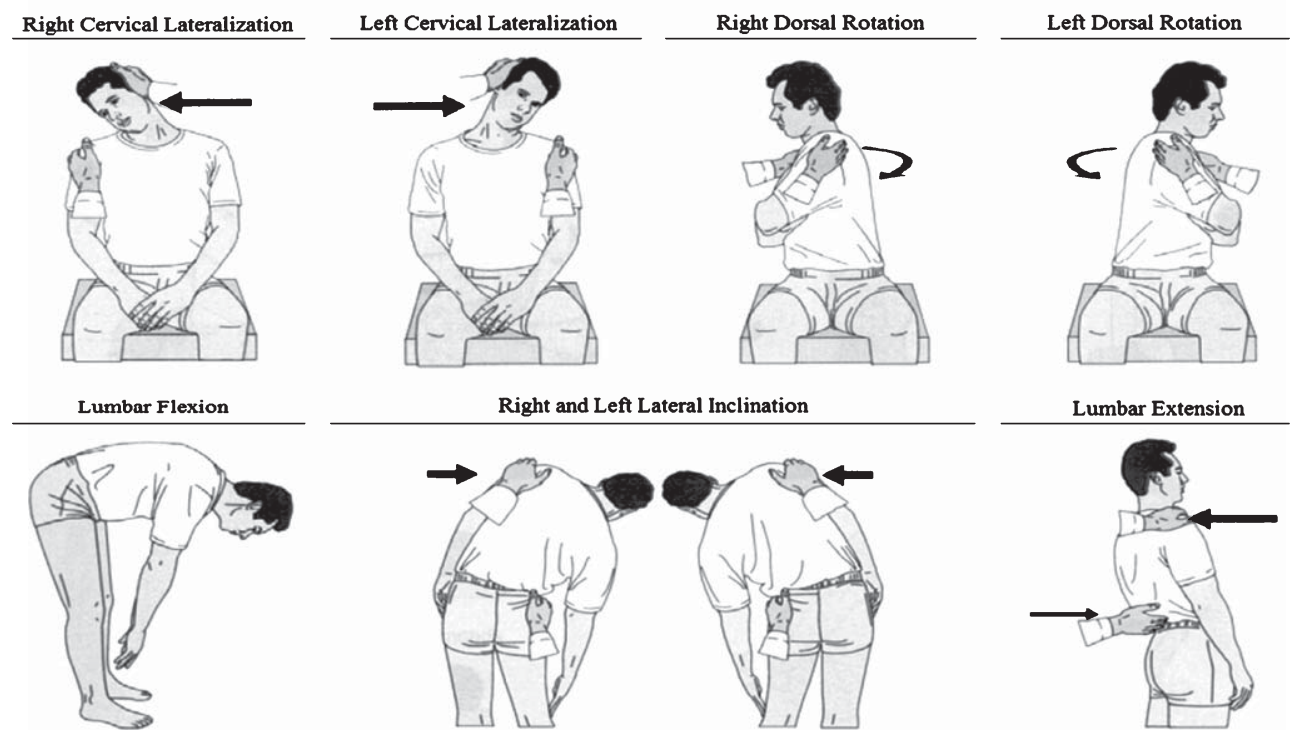

Fig. 4. Basic motions of a spine, [4].

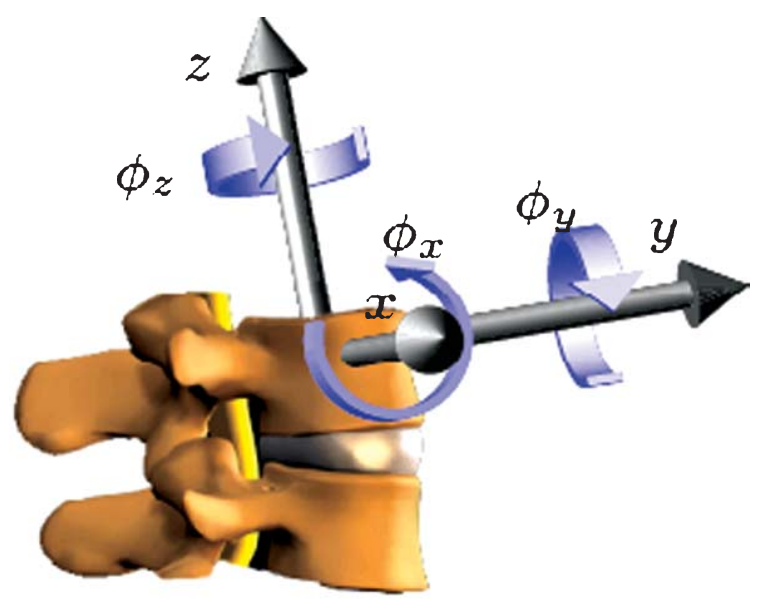

Fig. 5. Reference axes and rotations for motion of a vertebra.

stiffness, size variations and sub-structures that affect the spine is almost impossible. Several models have been attempted whose few significant examples are reported in the reference list.
Table 1

Range of the motions of the spine, [4]

\begin{tabular}{ccccc}
\hline & Flexion & Extension & Lateralization & Rotation \\
\hline $\begin{array}{c}\text { Cervical } \\
\text { Segment }\end{array}$ & $\begin{array}{c}\text { Ment in the } \\
\text { wishbone }\end{array}$ & $\begin{array}{c}\text { Ment } 18 \mathrm{~cm} \\
\text { of the } \\
\text { wishbone }\end{array}$ & 30 degrees & 60 degrees \\
Thoracic & - & - & 30 degrees & 75 degrees \\
$\begin{array}{c}\text { Segment } \\
\text { Lumbar } \\
\text { Segment }\end{array}$ & 60 degrees & 30 degrees & 20 degrees & 5 degrees \\
& & & &
\end{tabular}

In this paper a specific model is proposed for the lumbar part of a spine that is the one that mostly supports the weight of a body. A first simplifying assumption for a model of the spine has been applied by considering that all solicitations of the spine are vertically applied at the center of the vertebra.

Important characteristics for a proper spine model can be considered the mechanical properties of the intervertebral discs, like damping rate and stiffness; geometrical dimensions of the vertebras and discs; 
possible movements of each spine segment; and the load applied to the spine.

A real vertebra is composed of a body, an arch, and vertebral processes. Therefore, a human spine can be modeled as a multi-module parallel manipulator with a 3D design of the vertebras as the fixed and movable platforms of parallel manipulators.

The average circumference of the lower endplate of the fourth lumbar vertebra can be estimated as
$141 \mathrm{~mm}$ and the average surface area can be computed as $1492 \mathrm{~mm}^{2}$. Lumbar vertebras present an increasing pedicle width from a mean value of $9.6 \mathrm{~mm}$ at $\mathrm{L} 3$ to $16.2 \mathrm{~mm}$ at $\mathrm{L} 5$, as reported in [7].

In Fig. 6(a) a geometrical model is proposed with three bars in the platform as to represent the vertebral processes. In Fig. 6(b) the 3D SolidWorks model is shown for a general vertebra with characteristic angles among the vertebral processes.

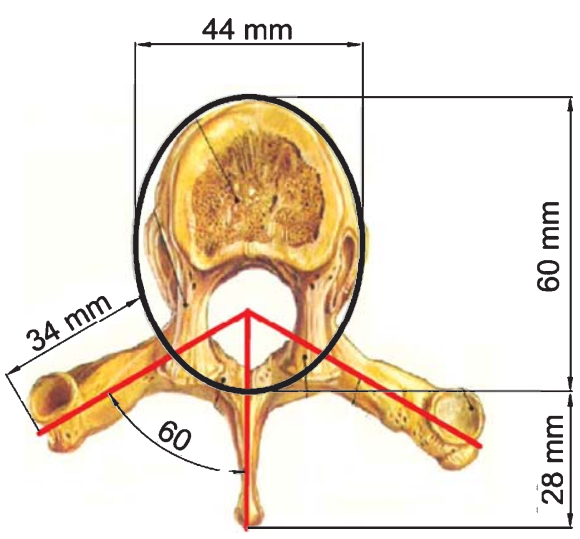

(a)

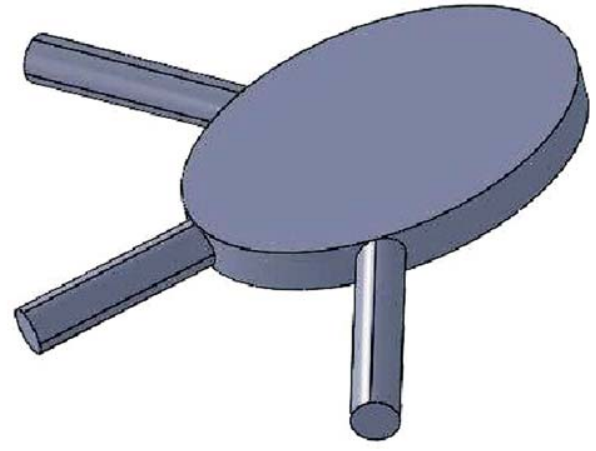

(b)

Fig. 6. Geometrical figure of a vertebra: (a) Average dimensions; (b) A CAD model.

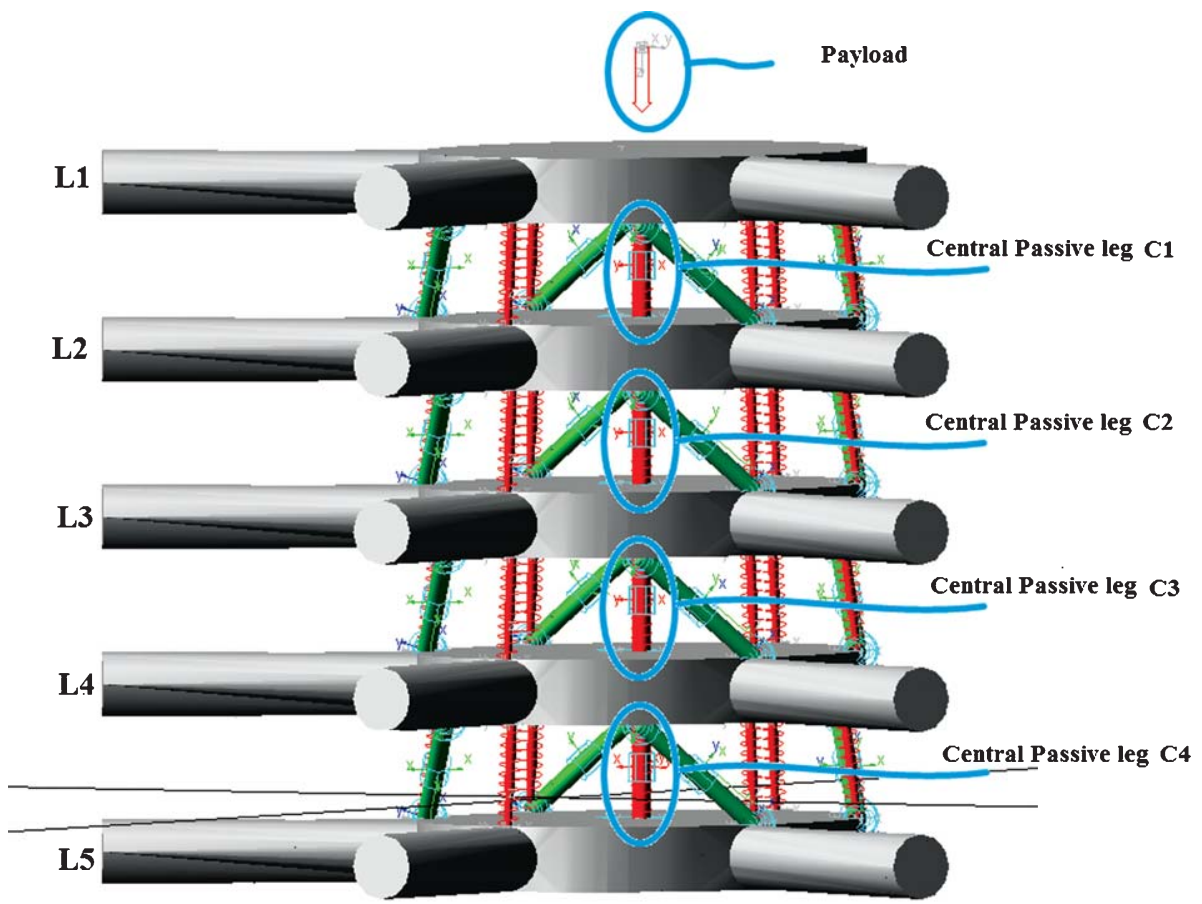

Fig. 7. A 3D ADAMS model with general parts identifications. 


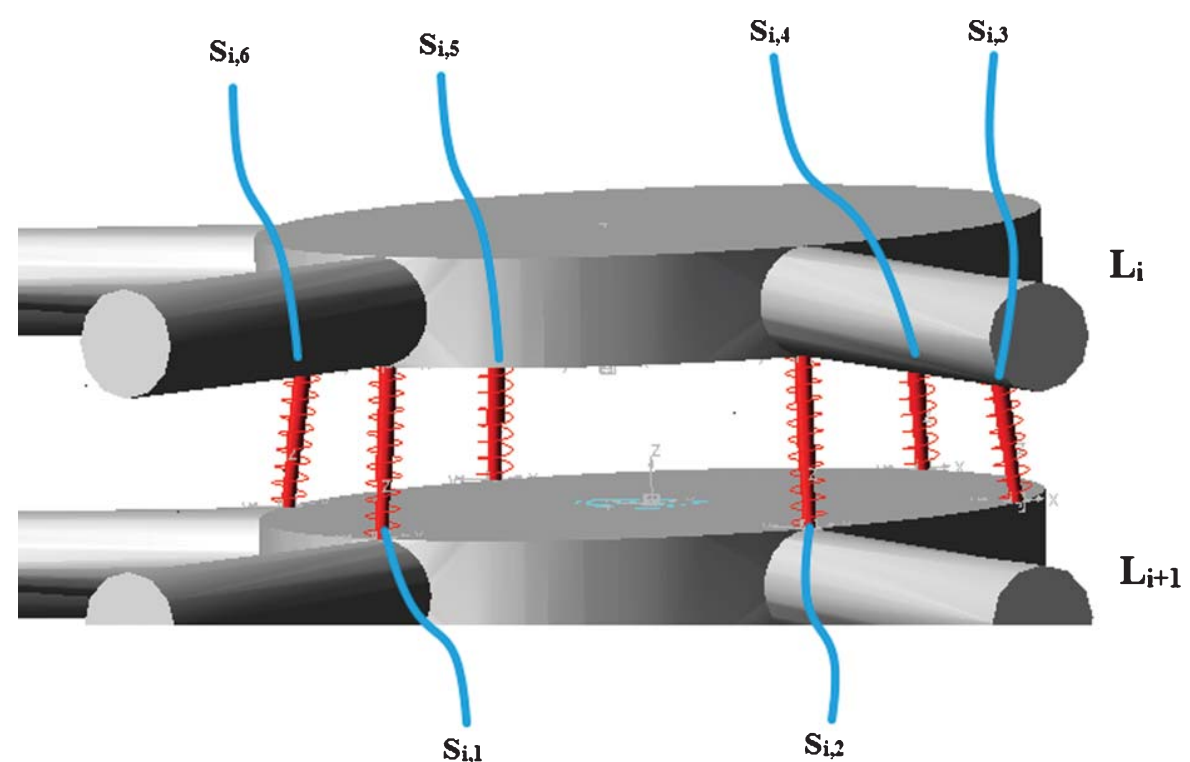

(a)

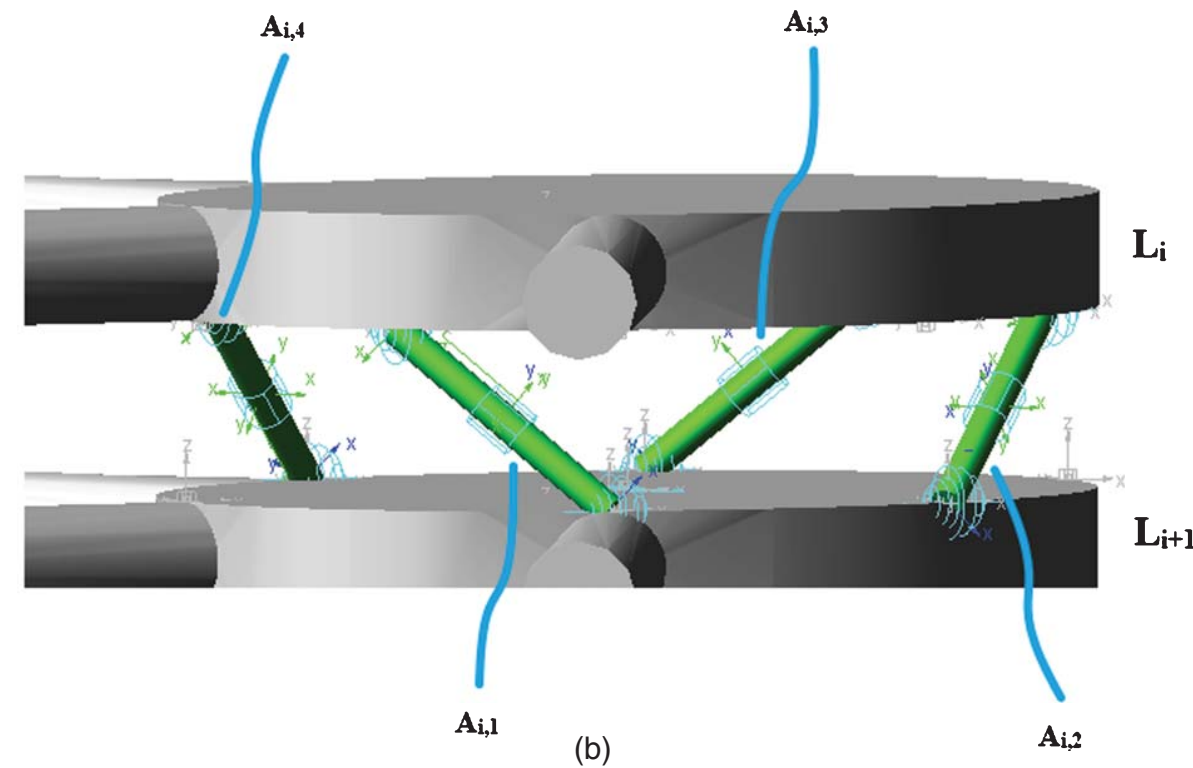

Fig. 8. The labels for part identification: (a) the springs; (b) the actuating legs.

The 3D model has been elaborated in SolidWorks and then has been exported to ADAMS with the aim to run a dynamic simulation for computing motion properties and reaction forces.

In ADAMS the discus has been modeled as a body that is attached to the assembled vertebras. In addition, springs have been considered to simulate the discus with a proper stiffness; actuating legs of the parallel manipulator structure have been assumed to simulate muscles and tendons; and finally a passive leg and the joints are also added to connect consecutively the vertebras and the other parts of the model. Thus, between each two vertebras the following components have been considered in the ADAMS mechanical model: seven spring links with damping behavior; four actuating legs; one passive leg; five cylindrical joints; and nine spherical joints. Each of these parts has specific functions as connecting other parts, simulating 
motions or simulating stiffness and damping. In addition, the proposed model can include curvature inputs for a spine as using suitable initial configurations and relative positions of the parallel manipulator units.

The vertebras in the form of platforms of parallel manipulator structures are named with the same medical names for real vertebras at the lumbar segment as L1, L2, L3, L4 and L5. In order to give a better understand of the model, the other parts are named as follows: the passive legs in the center of the platforms as $\mathrm{C} 1, \mathrm{C} 2, \mathrm{C} 3$ and $\mathrm{C} 4$; the springs in counterclockwise way as $S_{i, 1}, S_{i, 2}, S_{i, 3}, S_{i, 4}, S_{i, 5}, S_{i, 6}$; the actuating legs in counterclockwise way as $A_{i, 1}, A_{i, 2}, A_{i, 3}, A_{i, 4}$. In Figs. 7 and 8 the ADAMS model with the actuating legs, payload force and spring links are shown with their respective identifications.

The model for moving a platform as a vertebra has four actuating legs that give the movement for spine simulating tendons and muscles of a real spine. These legs are symmetrically located in the manipulator design, with a small slope of 5 degrees between each other with the aim to avoid singularities during numerical computations within the ADAMS software.

Each actuating leg is modeled through the following components: two spherical joints connecting the leg to the top and bottom vertebras, two bars simulating the actuator itself by sliding within each other, and one cylindrical joint connecting those two bars. An actuating leg with the active model is shown in detail in Fig. 9.

In order to have a correct attachment between two consecutive platforms, a passive leg is installed in the center of the platform. This passive leg is composed of one spherical joint, two bars, one cylindrical joint and one spring, similarly to active legs. The leg is assembled to block the translation motion between two

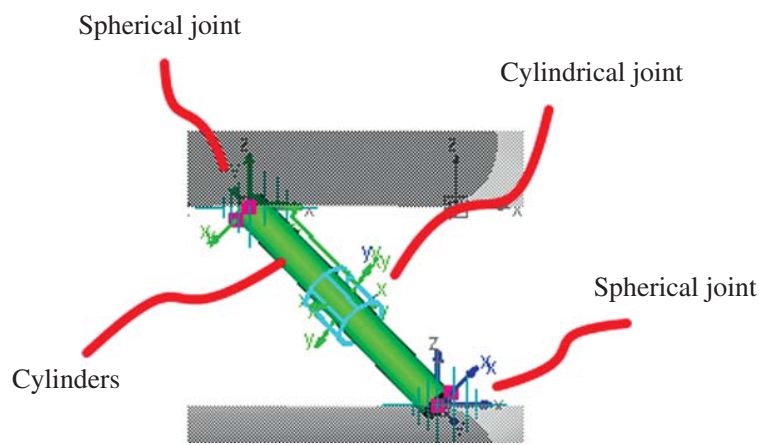

Fig. 9. Detail of the actuators model in a leg by two sliding bars. consecutives platforms along $\mathrm{X}$ and $\mathrm{Y}$ axes. In addition a spring is modeled as connecting the two platforms concentrically with the two cylinders in the passive leg. The passive leg with the above model is shown in Fig. 10.

As default in ADAMS software each spring has a damper attached with it. Therefore all the springs in the model have dampers concentrically inserted. In Table 2 the stiffness in axial direction of the intervertebral discs in a real spine is reported as indicated in [6].

Therefore, a model for a vertebra has seven spring links with damping behaviors symmetrically installed connecting two consecutive platforms. By using data in the Table 2 a model has been considered with an average stiffness of $1.5 \mathrm{MN} / \mathrm{m}$ that has been distributed over the seven springs. Thus, all the springs have a stiffness of $0.2 \mathrm{MN} / \mathrm{m}$, but the central spring of the passive leg has a stiffness of $0.3 \mathrm{MN} / \mathrm{m}$. This last spring has modeled the discus with its distributed stiffness and damping.

\section{Simulation results}

The above model has been tested with numerical simulation that have been used also to characterize the

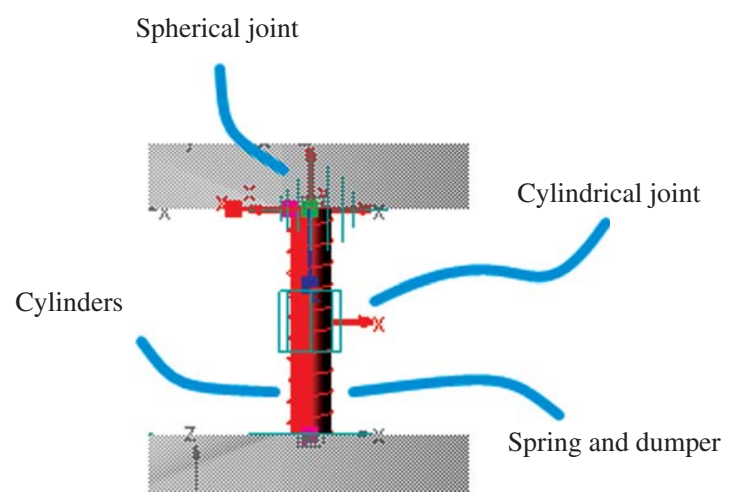

Fig. 10. Detail of the model for the passive central leg.

Table 2

Stiffness data of the intervertebral discs for the lumbar segment, [f6]

\begin{tabular}{lc}
\hline Vertebra & Stiffness [MN/m] \\
\hline L1 & 1,570 \\
L2 & 1,472 \\
L3 & 1,472 \\
L4 & 1,373 \\
L5 & 1,079 \\
\hline
\end{tabular}


reported simulated motions where the flexion of the lumbar spine model with a displacement of $1 \mathrm{~mm}$ corresponds to a rotation of 5 degrees in the respective axis of movement. A payload has been assumed with a step force of $300 \mathrm{~N}$ to approximately correspond to the mass of the human body that is supported by a spine. This force is considered as applied centrally to the first platform (L1) as shown in Fig. 7. For a simulation of the motion an actuating displacement of $1 \mathrm{~mm}$ has been imposed to actuators $A_{i, 1}$ and $A_{i, 4}$. The other two actuators are assumed with free motion in a way that does not interfere with the movement of the lumbar spine.

Illustrative numerical results are reported in Figs. 11 to 17 both to discuss the feasibility of the proposed model and to characterize numerically the biomechan- ics of the lumbar spine via a simulation through the mechanics of parallel manipulators.

In Fig. 11 the computed spring forces between the L1 and L2 vertebras are shown with a stiffness of $0.2 \mathrm{MN} / \mathrm{m}$ and a damping of $10 \mathrm{Ns} / \mathrm{m}$. Likewise, Fig. 12 shows the spring forces between the L4 and L5 vertebras for which a stiffness of $0.4 \mathrm{MN} / \mathrm{n}$ and a damping of $10 \mathrm{Ns} / \mathrm{m}$ have been assumed. Fig. 13 shows the reaction forces at the spherical joints that connect the actuators $A_{1,1}$ and $A_{1,4}$ to the platform L1. Similarly Fig. 14 shows the reaction forces at the spherical joints that connect the actuators $\mathrm{A}_{1,1}$ and $\mathrm{A}_{1,4}$ to the platform L2. Figure 15 shows the reaction forces at the spherical joints that connect the actuators $\mathrm{A}_{4,1}$ and $\mathrm{A}_{4,4}$ to the platform L4. Figure 16 shows the reaction forces

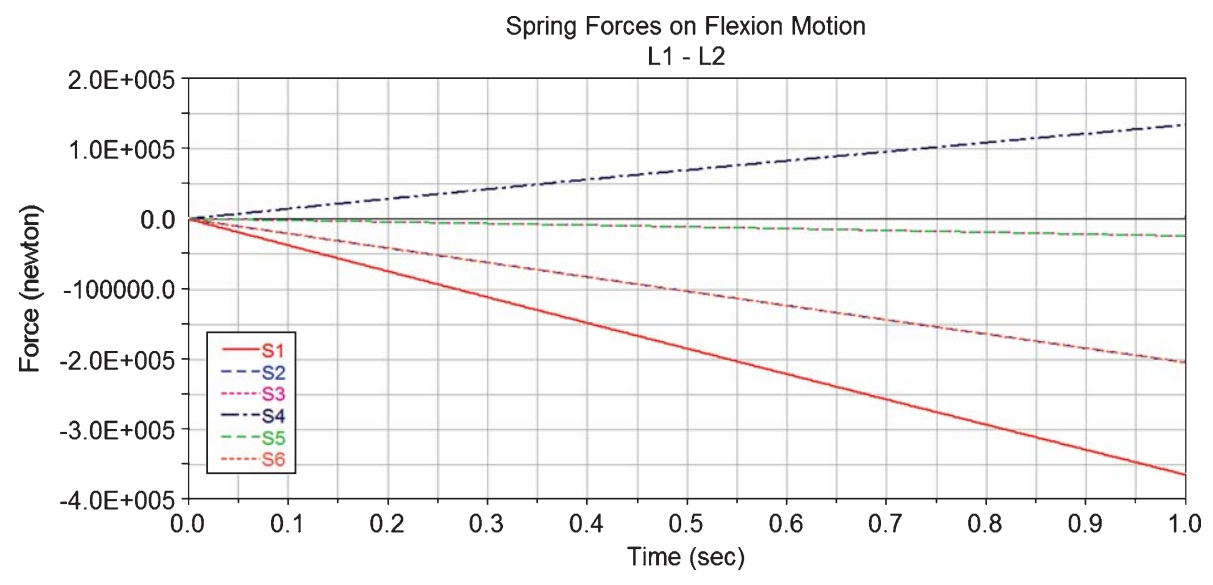

Fig. 11. Computed forces in $\mathrm{S}_{1, \mathrm{i}}$ springs for a simulated flexion of the lumbar spine segment.

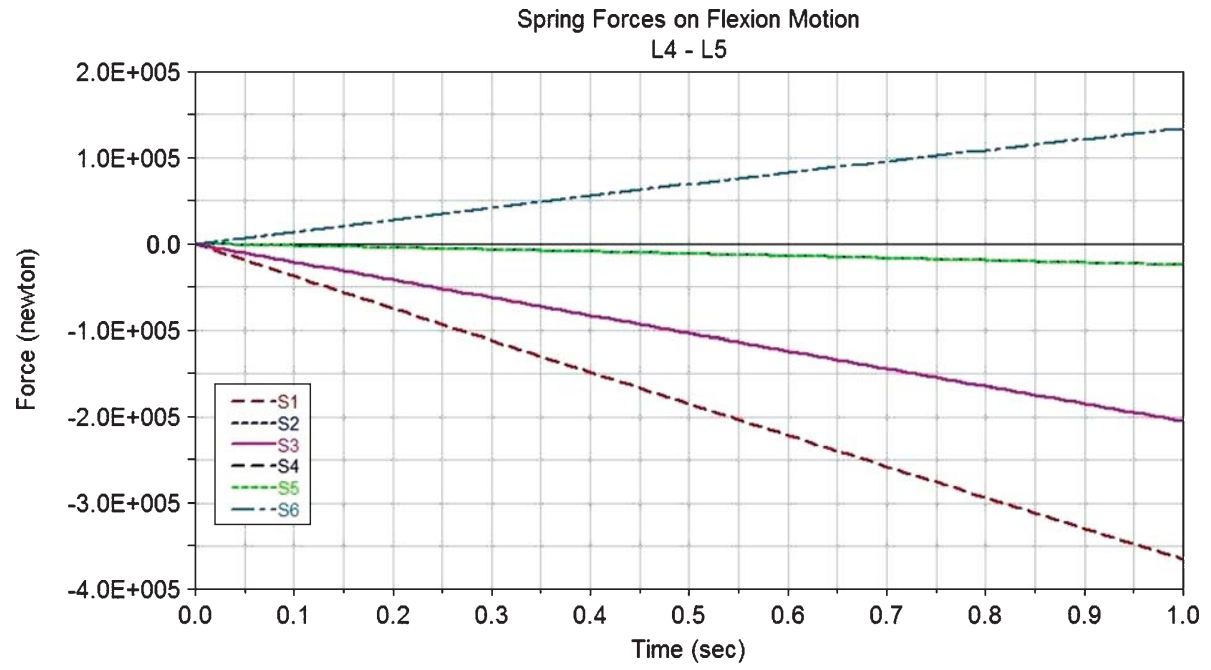

Fig. 12. Computed forces in $\mathrm{S}_{4, \mathrm{i}}$ springs for a simulated flexion of the lumbar spine segment. 
at the spherical joints that connect the actuators $\mathrm{A}_{4,1}$ and $\mathrm{A}_{4,4}$ to the platform L5. Finally, Fig. 17 shows the reaction forces at the springs and spherical joints of the passive legs $\mathrm{C} 1$ and $\mathrm{C} 4$.

It is to note that the reaction forces at the joints between the platforms L1 and L2 are larger than the reaction forces at the joints between L4 and L5. This properly simulates the human behavior although the springs are symmetrically installed to distribute the forces equally to each spring between each platform.

The reaction forces at the joints have a linear behavior because the imposed motion is linear and the modeled springs at the joints have a linear behavior too, since the intervertebral discs also presents a linear behavior with an elastic response. In addition the computed results for the spring forces are reasonable as compared with the values that are available in the literature for human spine, like those reported in Table 2.

Figures 11 to 17 shows a time history of the reaction forces that are applied for a predefined initial position of the spine model. The compatibility of results with literature enables to conclude that the proposed parallel structure can be successfully used to simulate a human spine at a macroscopic level by considering several conditions in terms of forces and/or displacements for a specific intervertebral disk or for distributed condi-

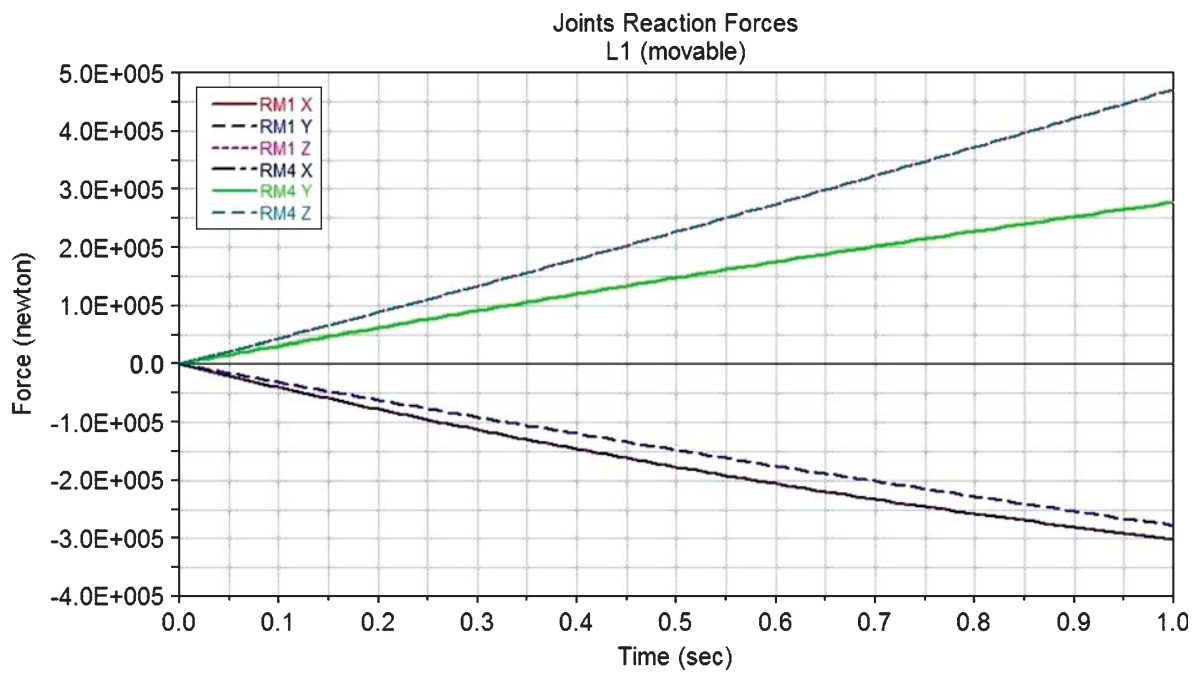

Fig. 13. Computed reaction forces at spherical joints that connects $\mathrm{L} 1-\mathrm{A}_{1,1}$ and $\mathrm{L} 1-\mathrm{A}_{1,4}$.

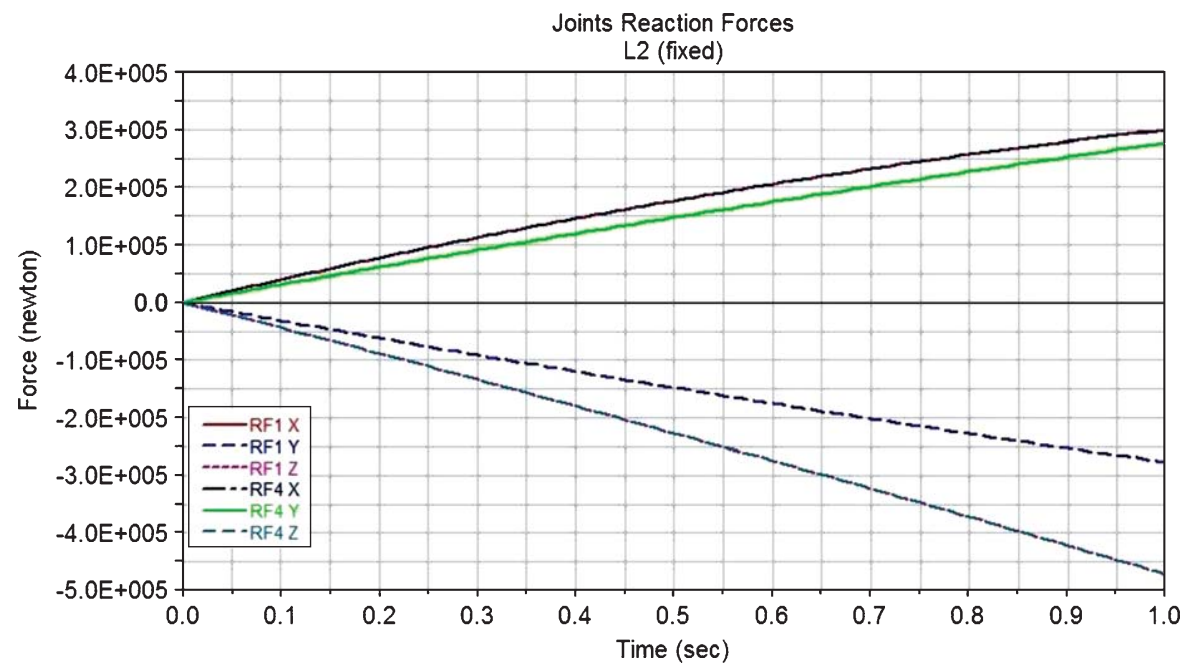

Fig. 14. Computed reaction forces at spherical joints that connects $L 2-A_{1,1}$ and $L 2-A_{1,4}$. 


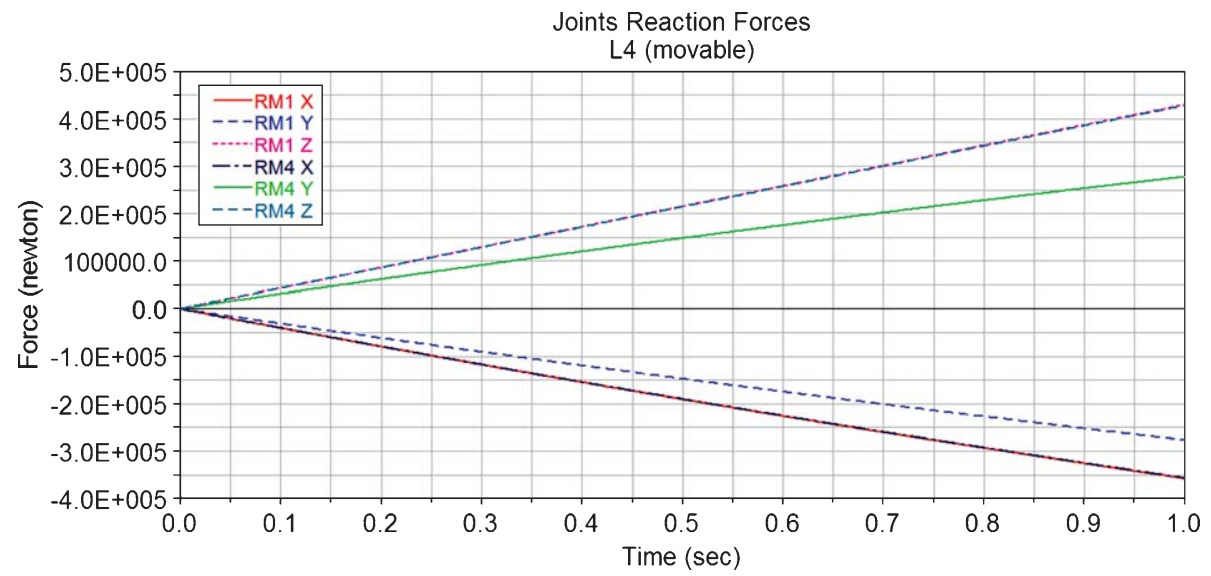

Fig. 15. Computed reaction forces at spherical joints that connects $\mathrm{L} 4-\mathrm{A}_{4,1}$ and $\mathrm{L} 4-\mathrm{A}_{4,4}$.

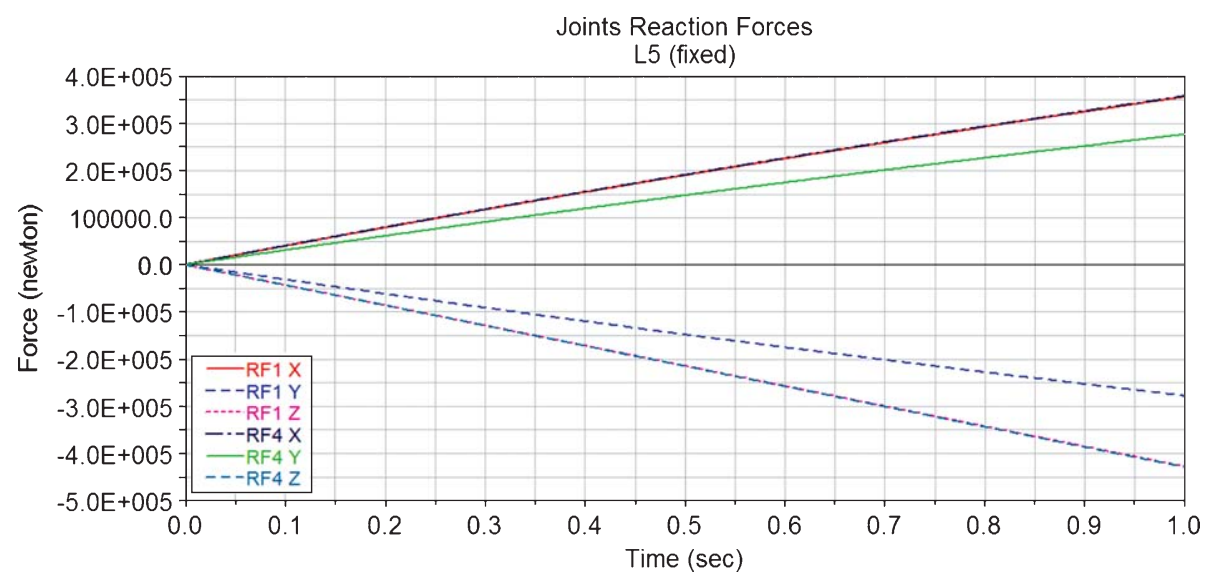

Fig. 16. Computed reaction forces at spherical joints that connects L5-A 4,1 and L5-A4,4.

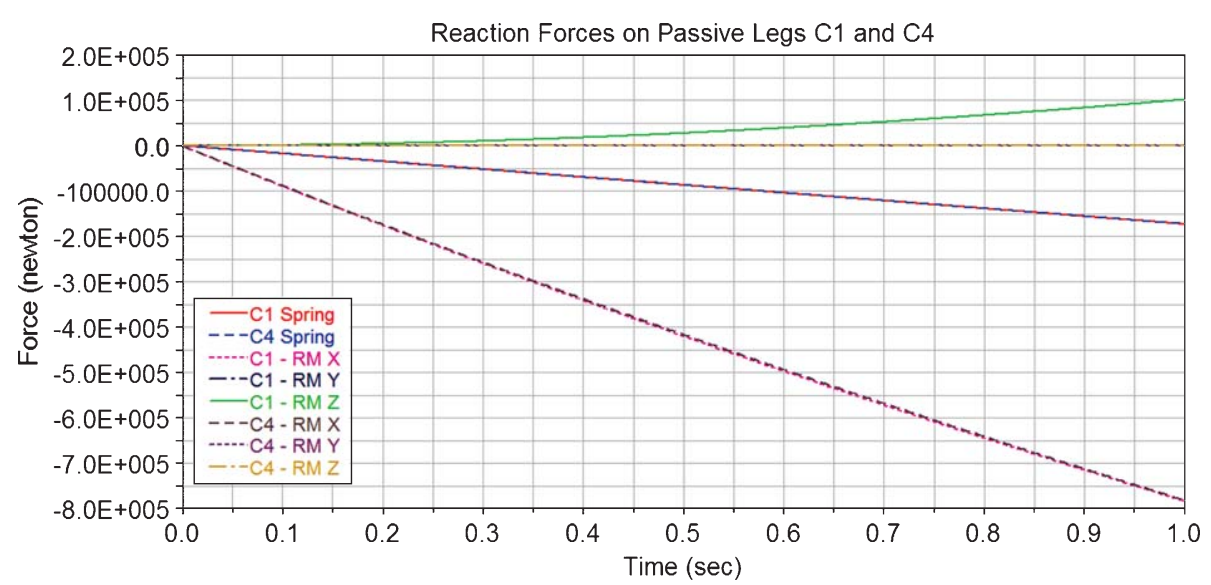

Fig. 17. Computed reaction forces in the Passive Legs $\mathrm{C} 1$ and $\mathrm{C} 4$. 
tions along a spine segment. Thus, several postures of a human spine can be simulated with proper input data in the proposed model with a direct physical interpretation by using computed reaction forces as output of a simulation.

\section{Conclusion}

In this paper, a model for the human lumbar spine is proposed for analyzing applied forces on the intervertebral discs through a suitable motion simulation. The proposed model has been elaborated by means of the SolidWorks software and then it has been simulated with the ADAMS software that provides a dynamic simulation for 3D models.

The contributions of this work can be considered as:

- A new model for the human spine, where the spine properties has been used as characteristics of a parallel manipulator with a suitable spring in order to properly simulate the smooth behavior of the intervertebral discs and actuation motions of muscles and tendons.

- Simulation results that for the springs and joints reaction forces in simulated human motions can give a suitable measure of the forces that intervertebral discs support during motions.

The general concepts for modeling and numerical simulation have been applied for the lumbar segment of the human spine, to show the feasibility and efficiency of the proposed models and computations. Another result both with the proposed model and presented work can be recognized in providing a virtual model that can be used even by doctors to study functionality of human spine preliminarily or as an aid in biomechanical study of torso functionality.

The authors will have not claimed that the proposed model is better of existing ones in the literature, but they will stress that is an interesting alternative way for considering parallel actions of the musco-skeleton system in a human spine. To the knowledge of the authors this is the first time that a parallel manipulators is used to model human spine. The current model can be indeed considered a preliminary conceptual design whose future enrichments can give better correspondence with human body, but again at functionality level mainly. This model has been indeed also an inspiration for a design of a human-like torso for humanoid robots as reported in [3].

\section{References}

[1] M. Ceccarelli, Fundamentals of Mechanics of Robotic Manipulation, Kluwer Academic Publishers, Dordrecht, 2004

[2] IFToMM, International Federation for the Promotion of Mechanism and Machine Science, Terminology. http://130.15.85.212/indexa.html, 2009.

[3] C. Liang and M. Ceccarelli, Design and Simulation a WaistTrunk System for a Humanoid Robot, In proceedings of the 18th CISM-IFToMM Symposium on Robot Design, Dynamics, and Control, Springer, Wien (2009), 217-225.

[4] J. Natour, Coluna Vertebral - Conhecimentos Básicos, Sociedade Brasileira de Reumatologia, Etcetera Ed., São Paulo, 2004, (In Portuguese).

[5] University of Maryland, A Patient's Guide to Anatomy and Function of the Spine, http://www.umm.edu/spinecenter/ education/anatomy_and_function_of_the_spine.htm, 2009.

[6] A. Vinciguerra, L. Aulisa and M. Ceccarelli, Stabilità e comportamento elastico del rachide, Minerva Ortopedica e Traumatologia 37(11) (1986), 717-723, (In Italian).

[7] S.H. Zhou, I.D. McCarthy, A.H. McGregor, R.R.H. Coombs and S.P.F. Hughes, Geometrical dimensions of the lower lumbar vertebrae - analysis of data from digitized CT images, Eur Spine Journal 9 (2000), 242-248.

[8] R. Cooper, C. Cardan and R. Allen, Computer visualisation of the moving human lumbar spine, Computers in Biology and Medicine 31(6) (2001), 451-469.

[9] H.J. Cramer, Y. King Liu and D.U. von Rosenberg, A distributed parameter model of the inertially loaded human spine, Journal of Biomechanics 9(3) (1976), 115-130.

[10] T. Pitzen, F. Geisler, D. Matthis, H. Müller-Storz, D. Barbier, W.I. Steudel and A. Feldges, A finite element model for predicting the biomechanical behaviour of the human lumbar spine, Control Engineering Practice 10(1) (2002), 83-90.

[11] J.F. Soechting and P.R. Paslay, A model for the human spine during impact including musculature influence, Journal of Biomechanics 6(2) (1973), 195-203.

[12] L. Nicholson, C. Maher, R. Adams and N. Phan-Thien, Stiffness properties of the human lumbar spine: A lumped parameter model, Clinical Biomechanics 16(4) (2001) 285 292.

[13] M.M. Panjabi, Three-dimensional mathematical model of the human spine structure, Journal of Biomechanics 6(6) (1973), 671-680.

[14] I.A.F. Stokes and M. Gardner-Morse, Lumbar spine maximum efforts and muscle recruitment patterns predicted by a model with multi-joint muscles and joints with stiffness, Journal of Biomechanics 28(2) (1995), 173-175.

[15] Y. Nakamura, K. Yamane, Y. Fujita and I. Suzuki, "Somatosensory Computation for Man-Machine Interface from Motion Capture Data and Musculoskeletal Human Model", IEEE Transactions on Robotics 21(1)(2005), pp. 58-66. 

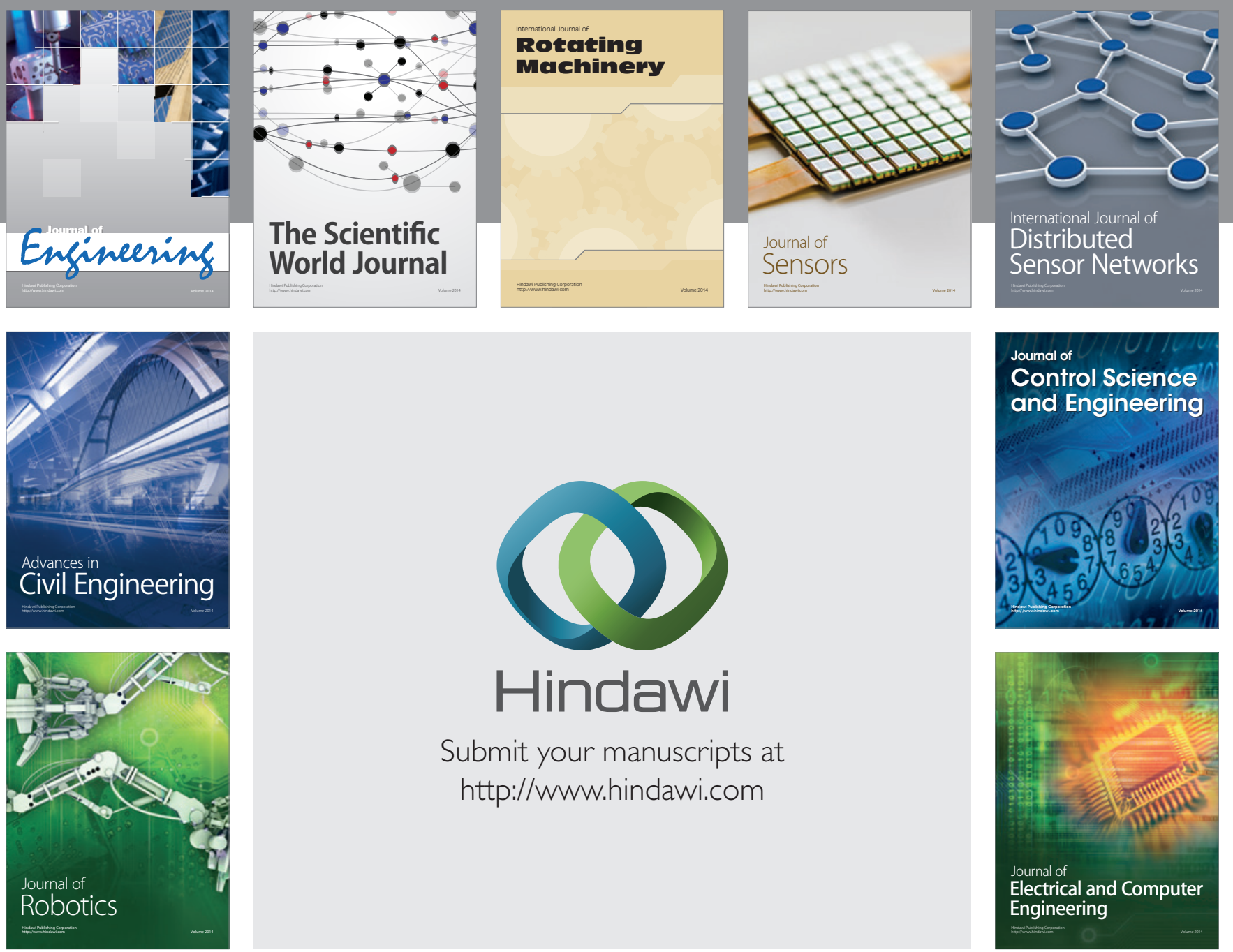

Submit your manuscripts at

http://www.hindawi.com
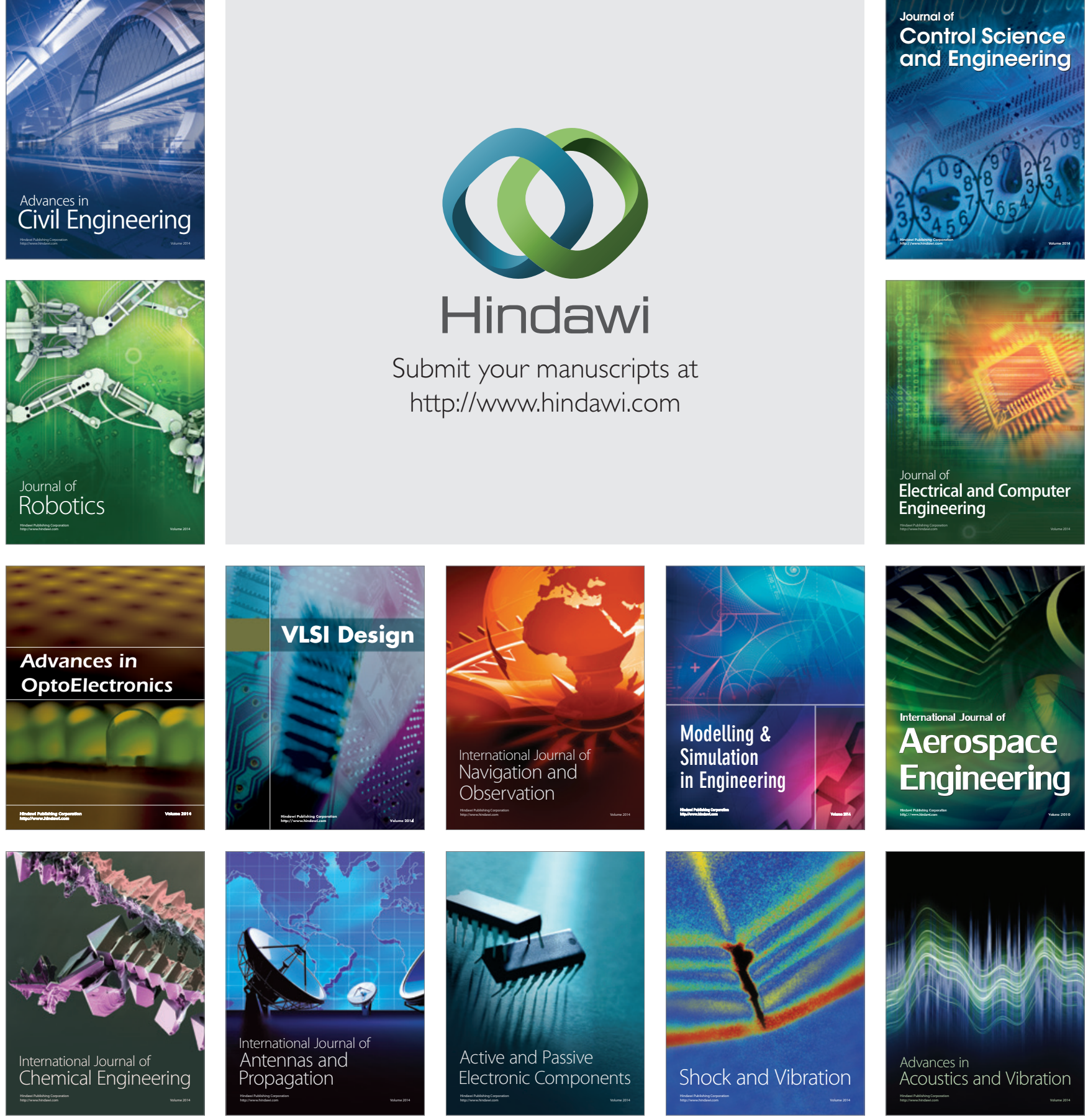